\title{
Ultraschall in der Therapie chronischer Wunden
}

\author{
Ultrasound in the Therapy of Chronic Wounds
}

Autoren

Institut
K. Klötgen, J. Klode, A. Körber, D. Schadendorf, J. Dissemond

Klinik und Poliklinik für Dermatologie, Venerologie und Allergologie, Universitätsklinikum Essen
Bibliografie

DoI $10.1055 / \mathrm{s}-0028-1119683$

Online-Publikation: 16.3.2009

Akt Dermatol 2009; 35:

237-242 @ Georg Thieme Verlag KG Stuttgart · New York ISSN 0340-2541

Korrespondenzadresse

Priv.-Doz. Dr. med.

Joachim Dissemond

Universitätsklinikum Essen

Klinik und Poliklinik für

Dermatologie, Venerologie und Allergologie

Hufelandstraße 55

45147 Essen

joachimdissemond@

hotmail.com

\section{Zusammenfassung \\ $\nabla$}

Ultraschall und insbesondere niederfrequenter Leistungsultraschall hat sich in der Konditionierung chronischer Wunden unterschiedlicher Genese als eine innovative und effektive Therapiealternative etablieren können. Die Handhabung der therapeutischen Ultraschallgeräte ist leicht erlernbar, der Einsatz sicher und ohne größeren Aufwand durchzuführen. Insbesondere durch die Induktion des Kavitationsphänomens kann Ultraschall therapeutisch sowohl für die Durchführung eines selektiven Débridements als auch für die Reduktion von Mikroorganismen einge-

\section{Einleitung \\ $\nabla$}

Ultraschall wird in der Medizin seit mehr als 70 Jahren für therapeutische und diagnostische Zwecke verwendet. Es können hierbei verschiedene qualitative Formen differenziert werden (๑ Tab. 1).

Für therapeutische Anwendungen wurde initial überwiegend hochfrequenter Ultraschall eingesetzt. Seit ca. 50 Jahren wird aber auch über den erfolgreichen Einsatz von niederfrequentem Ultraschall in der Konditionierung chronischer Wunden berichtet [1]. Für die Erzeugung des niederfrequenten Ultraschalls nutzt man den reziproken piezoelektrischen Effekt. Hierbei wird durch das Anlegen einer sinusförmigen Wechselspannung an ein im Schallkopf befindliches piezoelektrisches Material wie beispielsweise Bariumtitanat dieses durch seine physikalischen Eigenschaften in mechanische Schwingung versetzt und schließlich Energie erzeugt. Dieser Effekt wird auch als elektro-mechanischer Wandler bzw. Transducer bezeichnet [2]. Ultraschall besteht folglich aus mechanischen Wellen, deren physiologische Effekte das Ergebnis der Wechselwirkungen mit dem Transmissionsmedium sind. setzt werden. Eine Förderung der Granulation und die sich daraus potenziell ergebende raschere Wundheilung können trotz positiver Tendenzen mit den bislang vorliegenden wissenschaftlichen Daten im Sinne einer evidenzbasierten Medizin noch nicht endgültig bestätigt werden. Insgesamt betrachtet bietet der Einsatz von Ultraschall eine interessante Therapieoption in der Wundbehandlung, die bislang noch wenig genutzt wird. Zudem gibt es bislang noch zu wenige größere klinische Studien, die wissenschaftlich belegen, wann und bei welchen Indikationen der Ultraschall eingesetzt werden sollte.

Ein Teil der Ultraschallenergie wird bei der Anwendung in einem Gewebe absorbiert und in Wärme umgewandelt.

Die Wirkmechanismen von Ultraschall werden insbesondere durch dessen Frequenz bestimmt. Hohe Frequenzen erzeugen kurze Ultraschallwellen mit einer geringeren Eindringtiefe wohingegen niedrige Frequenzen lange Ultraschallwellen mit größerer Eindringtiefe generieren [3]. Abhängig von den jeweiligen physikalischen Kenngrößen Schallfrequenz, Schallwechseldruck, Wellenlänge und Schallstrahlungsdruck kommt es abhängig von der Reflexion und Absorption zu einer Umsetzung der Ultraschallenergie im Gewebe. Unterschiedliche Gewebe haben dabei unterschiedliche Absorptionsvermögen. So zeigen beispielsweise knöcherne Strukturen ein hohes Absorptionsvermögen, wohingegen dieses in fetthaltigen Geweben gering ist. Zudem werden Energieverlauf und Temperaturprofil des Ultraschalls im Gewebe durch die Reflexion an den Grenzflächen unterschiedlicher Medien bestimmt $[4,5]$. Im Gegensatz zu dem hochfrequenten Ultraschall besitzt der niederfrequente Ultraschall eine sehr lange Ultraschallwelle. Der Ultraschall niederfrequenter Bereiche kann somit 


\begin{tabular}{|c|c|c|c|}
\hline Art & Frequenz & Intensität/Leistung & Anwendung \\
\hline $\begin{array}{l}\text { niederfrequenter } \\
\text { Ultraschall }\end{array}$ & $30-120 \mathrm{kHz}$ & $0,05-1,5 \mathrm{~W} / \mathrm{cm}^{2}$ & Wundbehandlung \\
\hline $\begin{array}{l}\text { niederfrequenter } \\
\text { Leistungsultraschall }\end{array}$ & $20-60 \mathrm{kHz}$ & bis $200 \mathrm{~W} / \mathrm{cm}^{2}$ & $\begin{array}{l}\text { Wundbehandlung, } \\
\text { Liposuction, Thrombolyse } \\
\text { Zahnmedizin }\end{array}$ \\
\hline $\begin{array}{l}\text { hochfrequenter } \\
\text { Ultraschall }\end{array}$ & $0,8-5 \mathrm{MHz}$ & $0,1-3 \mathrm{~W} / \mathrm{cm}^{2}$ & $\begin{array}{l}\text { Physiotherapie, Kosmetik } \\
\text { Neurologie, Orthopädie }\end{array}$ \\
\hline $\begin{array}{l}\text { hochfrequenter } \\
\text { Leistungsultraschall }\end{array}$ & $5-10 \mathrm{MHz}$ & bis $1500 \mathrm{~W} / \mathrm{cm}^{2}$ & Tumortherapie, Chirurgie \\
\hline
\end{tabular}

Tab. 1 Qualitative Formen des therapeutischen Ultraschalls. niedrige Frequenzen mit enorm hoher Intensität nutzen und wird dann bei dem Auftreffen auf ein Medium teilweise reflektiert, ohne wesentliche thermische Effekte zu vermitteln.

\section{Geräte \\ $\nabla$}

Für die Wundgrundkonditionierung wird in einer modernen phasenadaptierten Versorgung chronischer Wunden überwiegend niederfrequenter Ultraschall beziehungsweise Leistungsultraschall eingesetzt. Aktuell befinden sich zwei unterschiedliche Konzepte von Geräten auf dem deutschen Markt, die bei unterschiedlichen Indikationen unterschiedliche Applikationsformen in der Wundtherapie ermöglichen. Die zunächst entwickelte Geräteart besteht aus einem Ultraschallgenerator und einer Sonde, die über einen Stab an den zu therapierenden Bereich aufgebracht werden kann. Eine stufenlose Regulation der Intensität ist orientiert anhand des Befundes und der Schmerzsymptomatik des Patienten möglich. Die Applikation des kontinuierlich oder gepulst ausgelösten niederfrequenten Ultraschalls erfolgt über einen angekoppelten Schallkopf direkt oder indirekt am Wirkungsort. Eine direkte Beschallung ist über eine suprakutan angebrachte Sonde möglich. Hierbei erfolgt die Behandlung beispielsweise für jeweils etwa 20 Minuten durch einen mit Ultraschall-Gel bedeckten sterilen Hydrogel- oder Hydrokolloidverband. Ein praktischer Vorteil ist, dass der Verband nach Abschluss des jeweiligen Therapieintervalls auf der Wunde belassen werden kann.

Die indirekte Anwendung erfolgt in einem Wasserbad. Dabei wird die Ultraschallsonde in einem Abstand von wenigen Zentimetern zu der Wundoberfläche gehalten. Die Therapieintervalle sollten jeweils etwa 10-20 Minuten betragen und möglichst täglich über einen Zeitraum von mindestens 2-4 Wochen durchgeführt werden. Da die Anwendung von Leitungswasser in der Wundtherapie heute sehr kritisch zu sehen ist, sollte entweder die Verwendung von Sterilfiltern bei Leitungswasser oder der Zusatz von nicht-zytotoxischen Antiseptika wie Octenidin oder Polihexanid empfohlen werden. Diese Geräte eignen sich aufgrund der einfachen Handhabung auch für die Anwendung durch geschulte Patienten oder Pflegepersonal.

Als neuere Alternative wurde vor mehreren Jahren ein sogenannter Ultraschall-Dissektor entwickelt ( $\bullet$ Abb. 1).

Der Ultraschall-Dissektor ermöglicht die Ankopplung von niederfrequentem Leistungsultraschall mit einer Arbeitsfrequenz von $25 \mathrm{kHz}$ und einer Leistung von $35-40 \mathrm{~W} / \mathrm{cm}^{2}$ über eine in ein Handstück eingearbeitete Sonde. Bei dieser sehr effektiven Methode ist aufgrund der Schmerzhaftigkeit auf eine ausreichende Analgesie zu achten. Oft reicht allerdings bereits die Applikation eines topischen Lokalanästhetikums in Form einer Creme für einen Zeitraum von 30-60 Minuten vor Intervention

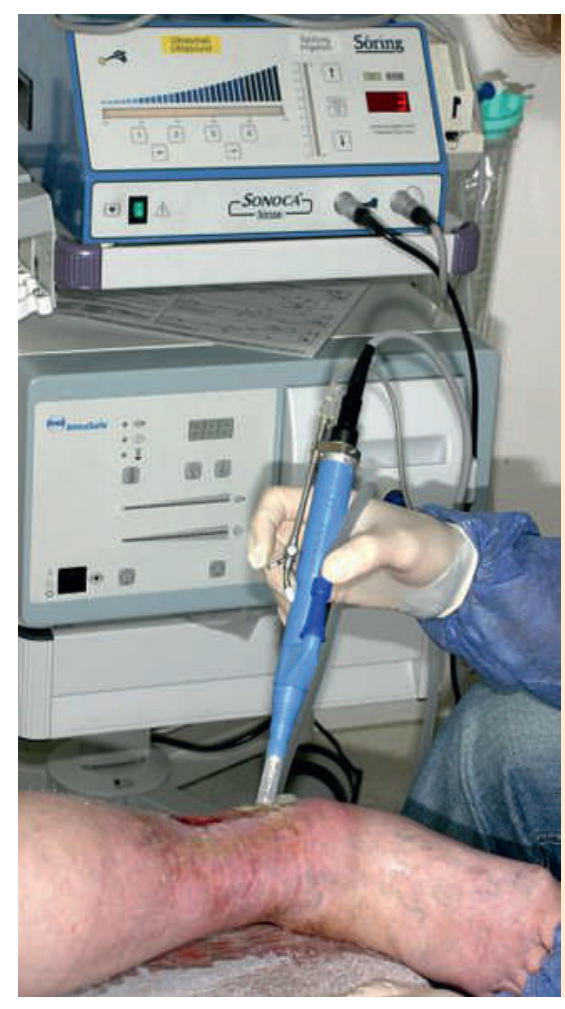

Abb. 1 Praktische Durchführung der Wundsäuberung eines Ulcus cruris mit niederfrequentem Leistungsultraschall.

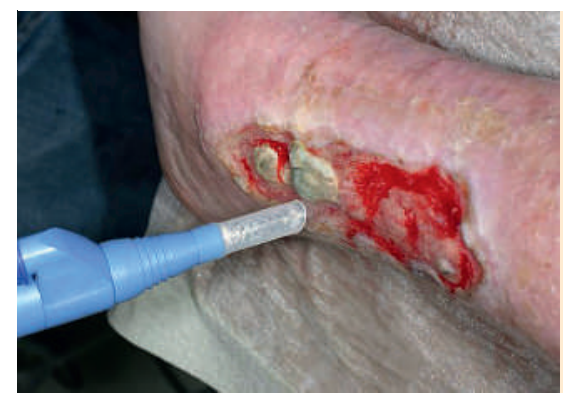

Abb. 2 An dem kaudalen Anteil des Ulcus cruris wurden bereits mittels niederfrequentem Leistungsultraschall Nekrosen und Fibrin abgetragen.

aus. Über die Spülflüssigkeit, die primär der Kopplung des Ultraschalls dient und zentral durch das Handstück fließt, können Zusätze in die Wunden eingebracht werden. Zudem befindet sich in dem Handstück eine Absaugvorrichtung, die entstehende Aerosole zu einem großen Teil aufsaugen soll um eine Kontamination der Umgebung zu verhindern. Bei dem Ultraschall-Dissektor handelt es sich um ein kompaktes mobiles Gerät, das ausschließlich durch geschulte Ärzte angewendet werden sollte (॰ Abb. 2). 


\section{Wundgrundkonditionierung \\ $\nabla$}

Unter dem Begriff „Wundgrundkonditionierung“ oder „wound bed preparation“ werden alle Maßnahmen zusammengefasst, die im Rahmen einer phasenadaptierten Wundbehandlung einen möglichst raschen Wundverschluss vorbereiten. Somit muss nach einem vollständigen Débridement insbesondere eine Förderung der Ausbildung von Granulationsgewebe erfolgen.

Die Vermittlung der Wirkungen von Ultraschall in chronischen Wunden lassen sich in mechanische Einwirkungen, insbesondere auf die Gewebeoberflächen, mechano-akustische/bio-akustische Effekte, insbesondere auf Mikroorganismen, und thermische und nicht-thermische Einflüsse, insbesondere auf tiefere Gewebeschichten, unterteilen [6]. Da hochfrequenter Ultraschall überwiegend thermische Effekte vermittelt und ausgehend von der primären physikalischen Energieform als Mechanotherapie gesehen werden kann, hat er sich lediglich bei der Behandlung der Wundumgebung als geeignet erwiesen [6-8]. Als ein wesentlicher Mechanismus in der Vermittlung der Wirkungen von Ultraschall in der Wundbehandlung ist das Phänomen der Kavitation zu sehen. Die Kavitation beschreibt die Ausbildung kleinster Bläschen in flüssigen Medien durch Druck-Zug-Kräfte, die oszillieren (stabile Kavitation) und/oder implodieren (transiente Kavitation). Insbesondere durch die Implosion resultieren Mikroströmungen und Druckgradienten. Die Viskosität des Mediums und die Ultraschallintensität bestimmen das Ausmaß der Kavitationsbildung. Die Intensität muss für die Induktion der Kavitation einen Schwellenwert überschreiten. Bei Wassertemperaturen zwischen $32^{\circ} \mathrm{C}$ und $38^{\circ} \mathrm{C}$ genügt bereits bei beispielsweise $40 \mathrm{kHz}$ eine Intensität von etwa $100-150 \mathrm{~mW} / \mathrm{cm}^{2}$, wohingegen bei $1 \mathrm{MHz}$ die Intensität mehr als 10-fach größer sein muss [6]. Höhere Frequenzen erfordern somit höhere Intensitäten für die Induktion von Kavitation.

\section{Débridement}

Ein effektives Débridement chronischer Wunden wird durch die Behandlung mittels niederfrequenten Ultraschalls über verschiedene Mechanismen vermittelt ( $\bullet$ Tab. 2).

Die mechanische Abtragung avitaler Bestandteile und die Induktion einer weitgehend selektiven Nekrosektomie können hierbei als wesentliche Aspekte gesehen werden [9]. So konnte auch in einem Rattenmodell gezeigt werden, dass Ultraschallanwendung für jeweils 5 Minuten mit einer Frequenz von $50 \mathrm{kHz}$ effektiv sowohl die Entfernung von avitalen Bestandteilen als auch die Elimination einer standardisierten bakteriellen Kontamination mit Staphylococcus aureus erzielt werden konnte [9].

Auch In-vitro-Untersuchungen mit dem Ultraschall-Dissektor belegen eine Korrelation der verwendeten Leistung und der induzierbaren Keimzahlreduktion. Während einer Behandlungsdauer von 60 Sekunden in hohen Leistungsbereichen von $80 \%-100 \%$ der maximalen Leistung zeigte sich eine Eliminationsrate von über $90 \%$ für Streptokokken- und PseudomonasSpezies. In mittleren Leistungsbereichen von $60 \%$ der maximalen Leistung konnte proportional abhängig von der Einwirkdauer eine Eliminationsrate von sogar fast $100 \%$ für Streptokokken erzielt werden, wenn die Behandlungsdauer mindestens $2 \mathrm{Mi}-$ nuten betrug. Somit scheint die bakterizide Wirkung der Ultraschalltherapie sowohl von der Leistung als auch von der Behandlungsdauer abhängig zu sein [10-12]. Weitere In-vivo-Studien im Tiermodell zeigten in kontaminierten posttraumatischen Wunden sowohl eine effektive Reduktion der bakteriellen Besiedelung als auch eine Verringerung der Anzahl der nachfolgen-
Tab. 2 Mechanismen des Débridements.

Elimination avitaler Bestandteile, Nekrosektomie

Elimination von Mikroorganismen

Verstärkung der enzymatischen Fibrinolyse

Verstärkung der Wirkung von Antibiotika

Verstärkung der Wirkung von Antimykotika

Tab. 3 Mechanismen der Granulationsförderung.

Angioneogenese
Ausschüttung von Wachstumsfaktoren
Proliferation von Fibroblasten
Verbesserung der kutanen Vaskularisation

den Infektionen [13]. Weitere Untersuchungen belegen, dass die Wirksamkeit von Gentamycin in Kombination mit therapeutischem Ultraschall auf planktonische Bakterien ebenso wie auf Bakterien in Biofilmen signifikant erhöht werden konnte. Insgesamt zeigten sich Ultraschallbereiche der niedrigen Frequenzen ( $70 \mathrm{kHz}$ ) bezüglich ihrer Bakterieneliminationsraten signifikant effektiver wirksam verglichen mit hochfrequenten Ultraschallbereichen $(10 \mathrm{MHz}$ ) [11]. Es wurde von den Autoren diskutiert, dass der Ultraschall den Transport des topisch applizierten Antibiotikums in die Zellen erhöht [14]. Ein transient gesteigerter transzellulärer Transport hydrophiler Substanzen durch die gleichzeitige Applikation von Ultraschall wurde mehrfach beschrieben [15]. So konnte elektronenmikroskopisch gezeigt werden, dass durch die Kavitationswirkung Risse in der Zelloberfläche der Bakterien entstehen [16].

Einen weiteren potentiell relevanten Faktor in der Vermittlung des Débridements durch niederfrequenten Ultraschall kann in der Verstärkung der endogenen enzymatischen Fibrinolyse gesehen werden $[17,18]$. Sowohl in vitro als auch in mehreren Invivo-Modellen zeigte sich bei einer Ultraschalltherapie mit einer Frequenz von $0,5-1 \mathrm{MHz}$ und einer Intensität von mehr als $0,5 \mathrm{~W} / \mathrm{cm}^{2}$ eine deutlich erhöhte enzymatische Fibrinolyse, die als Autolyse ein Débridement unterstützen kann [19].

Der antibakterielle Effekt von therapeutischem Ultraschall scheint somit sowohl durch die Schädigung von Oberflächenstrukturen, Wirkungssteigerung von Antiseptika oder Antibiotika als auch über das Auftreten von transienten Öffnungen der Zellmembranen und der Induktion der enzymatischen Fibrinolyse vermittelt zu werden $[15,16,20]$. In der Literatur wird diese Synergie zwischen Ultraschall und Wirkstoff auch als „sonophoretischer Teilchentransport“ oder als „bioakustischer Effekt“ beschrieben [11].

\section{Granulation}

In der phasenadaptierten modernen Wundtherapie bildet die Förderung der Ausbildung von Granulationsgewebe die wesentliche Vorraussetzung für eine Re-Epithelisation ( Tab. 3).

Während der Ultraschalltherapie von Patienten mit einem Ulcus cruris venosum zeigten sich eine Erhöhung des $\mathrm{pO}_{2}$ und eine Verminderung des $\mathrm{pCO}_{2}$ des kutanen kapillären Blutflusses [21]. Eine Steigerung der kutanen Vaskularisation nach Applikation von niederfrequentem Ultraschall erbrachte einen um $25 \%$ erhöhten $\mathrm{pO}_{2}$ in $2 \mathrm{~cm}$ Gewebetiefe. Intrakutan konnte sogar eine bis zu $40 \%$ ige Steigerung des $\mathrm{pO}_{2}$ sowie eine Erhöhung der Temperatur um $3^{\circ} \mathrm{C}$ in $2 \mathrm{~cm}$ Gewebetiefe oder um $5^{\circ} \mathrm{C}$ intrakutan und schließlich eine Zunahme der kutanen Stoffwechselleistung beobachtet werden [7,22]. Als ursächlich für die Verbesserung 
der Mikrozirkulation wurde eine als „Mikromassage“ bezeichnete mechanische Reizung des Gefäßendothels und eine verstärkte Stickstoffmonoxid-Freisetzung diskutiert [22-24].

Sowohl durch hochfrequenten als auch durch niederfrequenten Ultraschall zeigte sich in vitro eine Steigerung der Proliferation in Fibroblasten, Monozyten und Osteoblasten sowie deren Proteinsynthese und Zytokinproduktion [4,25,26]. Bei Patienten mit einem Ulcus cruris venosum ergab eine adjuvante Therapie mit Ultraschall eine vermehrte Ausbildung von Granulationsgewebe, eine Stimulation der Protein- und Kollagensynthese sowie eine Stimulation der Fibroblasten und der Makrophagen [7]. Für die Wundgrundkonditionierung chronischer Wunden wurden für oberflächlich gelegene Wunden höhere Frequenzen, beispielsweise um $3 \mathrm{MHz}$, und für Wunden tieferer Gewebeschichten niedrigere Frequenzen, beispielsweise um $1 \mathrm{MHz}$, empfohlen [23].

Zusammenfassend soll durch den Einsatz von therapeutischem Ultraschall in der Wundgrundkonditionierung chronischer Wunden ein effektives Débridement, eine Verkürzung der inflammatorischen Phase und eine Beschleunigung der Granulation erreicht werden [4,27]. Von entscheidender Bedeutung für die therapeutische Wirksamkeit scheinen die Dosierungsparameter des Ultraschalls, Leistungsdichte in $\mathrm{W} / \mathrm{cm}^{2}$, Beschallungsart, Behandlungsdauer und die Applikationsfrequenz sowie die Gesamtzahl der Behandlungen zu sein $[4,28]$.

\section{Klinische Studien}

$\nabla$

Die Prävalenz, ein Ulcus cruris venosum als weltweit häufigste Art einer chronischen Wunde zu entwickeln, liegt bei etwa 1,5/1000 [29], ist höher für Frauen und steigt mit zunehmendem Lebensalter an $[29,30]$. Weiterhin sind es insbesondere Patienten mit einem Dekubitus und/oder einem diabetischen Fußsyndrom, die eine chronische Wunde entwickeln können [31]. Diese chronischen Wunden unterschiedlicher Genese zeigen oft einen therapierefraktären Verlauf, neigen zu Rezidiven und sind durch die notwendigen Verbandwechsel material- und pflegeaufwendig. Als wesentlicher Baustein in der Behandlung des Ulcus cruris venosum ist beispielsweise die Kompressionstherapie sowie die Verwendung verschiedener Wundauflagen zu nennen. Es kommen aber auch zunehmend physikalische Therapien wie Elektrostimulation, Vakuum-, Hydro- und Lasertherapien oder extrakorporale Stoßwellentherapien zum Einsatz. Ein gemeinsames Merkmal aller Methoden ist, dass deren Wirksamkeit in der Behandlung chronischer Wunden im Sinne einer evidenzbasierten Medizin nur unzureichend belegt werden konnte. Dies gilt auch für die Ultraschalltherapie chronischer Wunden. Die wenigen bislang publizierten klinischen Studien zu der Anwendung des therapeutischen Ultraschalls in der Behandlung von Patienten mit chronischen Wunden beschäftigen sich mit der Frage, wie diese Methode wirkt und wann sie eingesetzt werden sollte. Zudem wurde immer wieder versucht, zumindest Teilaspekte der vermittelten Effekte zu objektivieren. So berichteten beispielsweise Dyson et al. bereits 1976 und in den folgenden Jahrzehnten auch andere Arbeitsgruppen von einer Beschleunigung der Wundheilung durch die Anwendung von therapeutischem Ultraschall $[7,27,29,32]$. Dennoch wurde in einer 2000 veröffentlichten Meta-Analyse festgestellt, dass bezüglich der Beschleunigung der Wundheilung zumindest für das Ulcus cruris venosum durch die Therapie mit Ultraschall zwar Tendenzen aber keine signifikant verkürzten Abheilungsraten erzielt wer- den konnten [33]. Auch eine weitere Cochrane-Meta-Analyse über die Wundbehandlung von Patienten mit Druckulzerationen zeigte 2006 keinen signifikanten Vorteil durch den Einsatz einer Ultraschalltherapie. Es konnten in dieser Zusammenfassung jedoch auch lediglich 3 Studien mit insgesamt 146 Patienten ausgewertet werden. Zwei dieser Studien waren Plazebo-kontrolliert, in der dritten Studie wurde eine kombinierte Behandlung mit Ultraschall und ultraviolettem Licht mit Lasertherapie und einer Standardbehandlung verglichen. In keiner der 3 Studien konnte ein statistisch signifikanter Unterschied in der Heilungstendenz der Ulzerationen gesehen werden [34]. Der Studienvergleich wird allerdings durch die geringe Anzahl der eingeschlossenen Patienten, unterschiedliche Therapiekonzepte und Nachbeobachtungszeiträume erschwert. Auch in einer von Lundberg et al. publizierten Untersuchung zu der Abheilung venöser Ulzera mittels gepulsten Ultraschalls konnten keine signifikanten Effekte im Vergleich zu der Kontrollgruppe gefunden werden [35]. Dennoch existieren auch mehrere In-vivo-Studien, die eine Beschleunigung der Heilung von akuten und chronischen Wunden durch Ultraschallapplikation sowohl im Tiermodell als auch bei Patienten belegen [36-39]. Beispielsweise konnte in einer randomisierten Plazebo-kontrollierten Vergleichsstudie gezeigt werden, dass durch den Einsatz einer adjuvanten Therapie mit Ultraschall geringer Intensitäten wie beispielsweise $<0,5 \mathrm{~W} / \mathrm{cm}^{2}$ und einer Frequenz $<100 \mathrm{kHz}$ eine Beschleunigung der Abheilung bei Patienten mit einem Ulcus cruris venosum erzielt wird [7]. Niederfrequenter Ultraschall scheint zudem die Wirkung antibiotischer Therapien zu verstärken. So konnte in mehreren Studien ein gesteigerter Transport von Antibiotika in die Zellen sowohl bei planktonischen Kulturen als auch in vitro und in vivo Biofilmen nachgewiesen werden [11,14,28,40-45].

Eine 2008 veröffentlichte Cochrane-Meta-Analyse über therapeutischen Ultraschall In der Behandlung von Patienten mit venösen Ulzerationen vergleicht 8 klinische Studien [46]. Insgesamt 5 dieser Studien waren Plazebo-kontrolliert. Die 3 anderen Studien vergleichen niedrigfrequenten Ultraschall mit aktuellen Standardtherapien. Hervorzuheben ist in dieser Meta-Analyse die 2004 publizierte Untersuchung von Franek [47]. Hierbei wurden in 2 Untersuchungsarmen der klinische Effekt der Anwendung verschiedener Intensitäten des Ultraschalls (gepulst $1 \mathrm{MHz}$ mit 0,5 oder $1 \mathrm{~W} / \mathrm{cm}^{2}$ ) in einem $34^{\circ} \mathrm{C}$ warmen Wasserbad untersucht. In dem 3. untersuchten Vergleichsarm kam zudem eine ausschließliche, standardisierte, topische Behandlung zur Anwendung. Die besten Resultate konnten in der Behandlungsgruppe gefunden werden, die mit $0,5 \mathrm{~W} / \mathrm{cm}^{2}$ therapiert wurde. Interessanterweise waren die Resultate der Patienten in der Gruppe, die mit $1 \mathrm{~W} / \mathrm{cm}^{2}$ behandelt wurde, weniger effektiv in Bezug auf die wöchentliche Abheilungsrate und Volumenreduktion der Ulzera verglichen mit der Gruppe, die eine Standardtherapie ohne Ultraschall erhalten hatten.

In den anderen Plazebo-kontrollierten Studien konnten bezüglich der Abheilungsraten keine signifikanten Unterschiede zwischen der Verum- und der Plazebogruppen gefunden werden. Vergleicht man allerdings den Prozentsatz der abgeheilten Ulkusfläche, ergibt sich ein statistisch signifikanter Unterschied zugunsten der Gruppen, die tatsächlich mittels Ultraschall behandelt wurden.

Bei dem Vergleich der Studien, in denen Ultraschalltherapien mit Standardtherapien verglichen wurden, zeigt sich ebenfalls kein signifikanter Unterschied in Hinblick auf die Anzahl der abgeheilten Ulzera. Berücksichtigt man allerdings alle Studien zusammen, die Ultraschalltherapie mit Plazebotherapie und Stan- 
dardtherapie verglichen haben, kommt man sowohl zu einem statistisch signifikanten Unterschied bei der Anzahl der geheilten Ulzera als auch bei dem Prozentsatz der abgeheilten Ulkusfläche zugunsten der Therapie mittels Ultraschall. Einschränkend muss jedoch ergänzt werden, dass bei sämtlichen Studien eine schwache methodische Qualität mit einer geringen Anzahl von Patienten bestand.

Zusammenfassend kann festgehalten werden, dass die Studienlage zu der Wundbehandlung mit Ultraschall zwar auf positive Teilaspekte und Tendenzen hinweist, insgesamt betrachtet die Qualität der derzeit zur Verfügung stehenden Daten aber unzureichend ist. Meist fehlt die Vergleichbarkeit der eingesetzten Methoden, da mit Ultraschall verschiedener Intensitäten und Frequenzen über unterschiedliche Zeiträume bei unterschiedlichen Indikationen, Applikationsarten und untersuchten Endparametern berichtet wurde.

\section{Fazit}

Die Vorteile der Behandlung chronischer Wunden mittels therapeutischen Ultraschalls stellen die einfache Handhabung, die teilweise sogar von den Patienten übernommen werden kann, der sichere Umgang mit den Geräten, die Kombination mit anderen Wundheilungsmaßnahmen, der breite Anwendungsbereich, die gute Akzeptanz bei Anwendern und Patienten und die geringen Folgekosten dar. Wohingegen die regelmäßig notwendige Desinfektion der Geräte, die hohen Anschaffungskosten und der Zeitaufwand die Nachteile dieser Therapiemethode charakterisieren.

Unbestritten ist sicherlich, dass insbesondere der niederfrequente Leistungsultraschall eine sehr effektive Methode für die Durchführung eines Débridements darstellt. Ob allerdings durch therapeutisch eingesetzten Ultraschall auch eine Beschleunigung der Wundheilung chronischer Wunden erzielt werden kann, ist zumindest im Sinne einer evidenzbasierten Medizin abschließend nicht sicher zu bestätigen und erfordert weitere randomisierte und kontrollierte Studien.

\section{Abstract}

\section{Ultrasound in the Therapy of Chronic Wounds \\ V}

Ultrasound and especially low frequency ultrasound systems are innovative and effective alternative strategies for the treatment in wound bed preparation of different chronic wounds. The handling of the ultrasound systems is easy to learn, the use is safe and does not require much other equipment. The induction of the so-called cavitation-phenomena seems to be a major effect which permits the selective debridement and the reduction of microorganisms. Despite interesting tendencies in the promotion of granulation and thus faster overall wound healing there are only few well documented evidence-based data available so that no final statement can be made yet.

Putting it all together the use of ultrasound seems to be a not yet established but promising option in wound-treatment. But there still exists a lack of greater clinical trials, that scientifically underline when and in which indications ultrasound should be used.

\section{Literatur}

1 Nichter LS, Williams J. Ultrasonic wound debridement. J Hand Surg 1988; $13: 142-146$

2 Liebold K, Uhlemann C, Wollina U. Ulcus-Debridement mit niederfrequentem Ultraschall. ZfW 2001; 18: 20-22

3 Uhlemann C, Wollina U, Liebold K, Schreiber TU. Behandlung des Ulcus cruris venosum mit niederfrequentem Ultraschal (nf-US). Phys Med Rehab Kuor 2001; 11: 216-220

4 Dyson $M$, Preston $R$, Woledge $R$, Kitchen S. Longwave ultrasound. Physiotherapy 1999; 85: 40-49

5 Webster DF, Pond JB, Dyson M, Harvey W. The role of cavitation in the in vitro stimulation of protein synthesis in human fibroblasts by ultrasound. Ultrasound Med Biol 1978; 4: 343-351

6 Radandt RR. Niederfrequenter Ultraschall in der Wundheilung. Phys Med Rehab Kuror 2001; 11: 41 - 50

7 Peschen $M$, Weichenthal $M$, Schöpf E, Vanscheidt $W$. Low-frequency ultrasound treatment of chronic venous leg ulcers in an outpatient therapy. Acta Derm Venereol 1997; 77: 311 - 314

8 Hynynen $K$. The threshold for thermally significant cavitation in dog's thigh muscle in vivo. Ultrasound Med Biol 1991; 17: 157- 169

9 McDonald WS, Nichter LS. Debridement of bacterial and particulatecontaminated wounds. Ann Plast Surg 1994; 33: 142 - 147

10 Schulze CH, Oesser S, Hülskamp T, Thies E. Bakterizide Wirkung mobil applizierten Ultraschalls. Hefte Wundbehand 2001; 5: 29

11 Quian Z, Sagers RD, Pitt WG. The effect of ultrasonic frequency upon enhanced killing of Pseudomonas aeruginosa biofilms. Ann Biomed Eng 1997; 25: 69-76

12 Singer AJ, Coby CT, Singer AH, Thode HC, Tortora GT. The effects of lowfrequency ultrasound on Staphylococcus epidermis. Curr Microbiol 1999; 38: $194-196$

13 Nichter LS, McDonald WS, Gabriel K, Sloan GM, Reinisch JF. Efficacy of debridement and primary closure of contaminated wounds: a comparison of methods. Ann Plast Surg 1989; 23: 224-230

14 Pitt WG, McBride MO, Lunceford JK, Roper RJ, Sagers RD. Ultrasonic enhancement of antibiotic action on gram negative bacteria. Antimicrob Agents Chemother 1994; 38: 2577-2582

15 Mitragotri S, Blankschtein D, Langer $R$. Ultrasound-mediated transdermal protein delivery. Science 1995; 269: 850-851

16 Ukhov AY, Fedechko IM, Narepekha OM. Immunidikatoren bei der Behandlung infizierter Wunden mit niederfrequentem Ultraschall (russ.). Klin Khir 1990; $1: 10-12$

17 Francis CW, Onundarson PT, Carstensen EL et al. Enhancement of fibrinolysis by ultrasound. J Clin Invest 1992; 90: 2063-2068

18 Harpaz D, Chen X, Francis CW. Ultrasound enhancement of fibrinolysis and reperfusion in vitro. J Am Coll Cardiol 1993; 2: 1507-1511

19 Suchkova V, Siddiqi FN, Carstensen EL. Enhancement of fibrinolysis with 40-kHz ultrasound. Circulation 1998; 98: 1030-1035

20 Williams RG, Pitt WG. In vitro response of Escherichia coli to antibiotics and ultrasound at various insonation intensities. J Biomater Appl 1997; $12: 20-30$

21 Hartmann B, Drews B, Bassenge F. Wirkungen des Unterwasser-Ultraschalls auf (transkutanen) Sauerstoffpartialdruck und Abheilung venöser Ulzera. Phys Rehab Kur Med1994; 4: 143 - 144

22 Ottstadt B, Drews B, Hartmann B. Heilt subaqual applizierter niederfrequenter Ultraschall arteriell-venöse Ulcera cruris? Vasomed 1998; 10: $24-32$

23 Hart J. The use of ultrasound therapy in wound healing. J Wound Care 1998; $7: 25-28$

24 Knoch HG. Der niederfrequente Ultraschall als neue Therapiemöglichkeit in der Naturheilkunde. Ärztez Naturheilverf 1997; 38: $20-24$

25 Doan N, Reher P, Meghji S, Harris M. In vitro effects of therapeutic ultrasound on cell proliferation, protein synthesis, and cytokine production by human fibroblasts, osteoblasts, and monocytes. J Oral Maxillofac Surg 1999; 57: 409-419

26 Harvey W, Dyson M, Pond JB, Grahame R. The stimulation of protein synthesis in human fibroblasts by therapeutic ultrasound. Rheumatol Rehabil 1975; 14: 237

27 Dyson M, Franks C, Suckling J. Stimulation of healing of varicose ulcers by ultrasound. Ultrasonics 1976; 14: $232-236$

28 Johannsen F, Gam AN, Karlsmark T. Ultrasound therapy in chronic leg ulceration: a meta-analysis. Wound Repair Regen 1998; 6: 121-126

29 Callam MJ, Harper DR, Dale JJ, Ruckley CV, Prescott RJ. A controlled trial of weekly ultrasound therapy of chronic leg ulceration. Lancet 1987; 2: 204-206 
30 Margolis DJ, Bilker W, Knauss J, Baumgarten M, Strom BL. The incidence and prevalence of pressure ulcers among elderly patients in general medical practice. Ann Epidemiol 2002; 12: 321 - 325

31 Kaltenthaler E, Whitfield MD, Walters SJ, Akehurst RL, Paisley S. UK, USA and Canada: how do their pressure ulcer prevalence and incidence data compare? J Wound Care 2001; 10: 530-535

32 Ebenbichler G, Resch KL. Critical evaluation of ultrasound therapy. Wien Med Wochenschr 1994; 44: 51 - 53

33 Flemming K, Cullum N. Therapeutic ultrasound for venous leg ulcers. Cochrane Database Syst Rev 2000; 4: 1180

34 Baba-Akbari Sari A, Flemming K, Cullum NA, Wollina U. Therapeutic ultrasound for pressure ulcers. Cochrane Database Syst Rev 2006; 3: CD001275

35 Lundberg T, Nordsröm F, Brodda-Jansen G et al. Pulsed ultrasound does not improve healing of venous leg ulcers. Scand J Rehab Med 1990; 22: $195-197$

36 Speed CA. Therapeutic ultrasound in soft tissue lesions. Rheumatology 2001; 40: 1331 - 1336

37 Yang KH, Park SJ. Stimulation of fracture healing in a canine ulna fulldefect model by low-intensity pulsed ultrasound. Yonsei Med J 2001; 42: $503-508$

38 Tan J, Abisi S, Smith A, Burnand KG. A painless method of ultrasonically assisted debridement of chronic leg ulcers: a pilot study. Eur J Vasc Endovasc Surg 2007; 33 (2): 234-238
39 Kavros SJ, Schenck EC. Use of noncontact low-frequency ultrasound in the treatment of chronic foot and leg ulcerations: a 51-patient analysis. J Am Podiatr Med Assoc 2007; 97 (2): 95 - 101

40 Carmen JC, Nelson JL, Beckstead BL et al. Ultrasonic-enhanced gentamicin transport through colony biofilms of Pseudomonas aeruginosa and Escherichia coli. J Infect Chemother 2004; 10 (4): 193 - 199

41 Carmen JC, Roeder BL, Nelson JL et al. Treatment of biofilm infections on implants with low-frequency ultrasound and antibiotics. Am J Infect Control 2005; 33 (2): $78-82$

42 Runyan CM, Carmen JC, Beckstead BL et al. Low-frequency ultrasound increases outer membrane permeability of Pseudomonas aeruginosa. J Gen Appl Microbiol 2006; 52 (5): 295 - 301

43 Young SR, Dyson M. The effect of therapeutic ultrasound on angiogenesis. Ultrasound Med Biol 1990; 16: 809-816

44 Byl NN, McKenzie AL, West JM et al. Low-dose ultrasound effects on wound healing: a controlled study with Yucatan pigs. Arch Phys Med Rehabil 1992; 73: 656-664

45 Schabrun S, Chipchase L, Rickard H. Are therapeutic ultrasound units a potential vector for nosocomial infection? Physiother Res Int 2006; 11: $61-71$

46 Al-Kurdi D, Bell-Syer SE, Flemming K. Therapeutic ultrasound for venous leg ulcers. Cochrane Database Syst Rev 2008; 23: CD001180

47 Franek A, Chmielewska D, Brzezinska-Wcislo L, Slezak A, Blaszczak E. Application of various power densities of ultrasound in the treatment of leg ulcers. J Dermatolog Treat 2004; 15: 379-386 